\title{
Disordered Eating in Adolescents and Young Adults with Cystic Fibrosis
}

\author{
Alexandra Kass ${ }^{1}$, Laura Berbert ${ }^{2}$, Suzanne Dahlberg ${ }^{2}$, Elana Bern², Sabina Sabharwal ${ }^{3}$, \\ Jessica Leonard ${ }^{3}$, Tracy Richmond ${ }^{3}$, and Gregory Sawicki ${ }^{3}$ \\ ${ }^{1}$ Columbia University Irving Medical Center \\ ${ }^{2}$ Boston Children's Hospital \\ ${ }^{3}$ Children's Hospital Boston
}

February 10, 2022

\begin{abstract}
Introduction: There is evidence for increased risk of eating disorders in individuals with diet-treated chronic illnesses, however, data in patients with Cystic Fibrosis (CF) is less clear. No studies have evaluated avoidant/restrictive food intake disorder (ARFID) in the CF population. We investigated the prevalence of disordered eating, including ARFID, in adolescents and young adults with CF. Methods: Patients with CF aged 14-35 years were recruited to complete three validated surveys: (1)Eating Disorder Examination Questionnaire (EDE-Q), (2)Nine-Item Avoidant/Restrictive Food Intake Disorder Scale (NIAS), and (3)Cystic Fibrosis Questionnaire-Revised (CFQ-R). Univariate linear regression analysis identified baseline risk factors associated with these survey scores. Variables with univariate $\mathrm{p}<0.20$ were considered for inclusion in a multivariable linear regression model. Backwards stepwise linear regression was used to identify the final model. Results: A total of 52 patients enrolled. The prevalence of eating disorder measured on the EDE-Q was 9.6\%, and on the NIAS was $13.5 \%$. The CFQ-R eating and weight subscales were associated with scores on the EDE-Q, and CFQ-R eating subscale and being dF508 homozygous were correlated with the NIAS total score. Discussion: A clinically significant number of participants screened positive for disordered eating on the EDE-Q and NIAS. Scores on the eating and weight scales of the CFQ-R were associated with the scores on these surveys. Further work is needed to better understand the optimal way to use such tools to screen and treat for eating disorders in individuals with $\mathrm{CF}$.
\end{abstract}

\section{Hosted file}

CFED Patient main.docx available at https://authorea.com/users/459756/articles/555909disordered-eating-in-adolescents-and-young-adults-with-cystic-fibrosis

\section{Hosted file}

CFED Patient Tables.docx available at https://authorea.com/users/459756/articles/555909disordered-eating-in-adolescents-and-young-adults-with-cystic-fibrosis 